\section{Serological profile of hepatitis $B$ in children after the intro- duction of its vaccination in Burkina Faso}

\author{
Makoura Barro, ${ }^{1}$ Diane Valea, ${ }^{2}$ \\ Saga Alain Ouermi, ${ }^{3}$ \\ Sanata Sessouma, ${ }^{2}$ Bintou Sanogo, ${ }^{1}$ \\ Ibrahim Ad Bafa Ouattara,1 \\ Abdoul Salam Ouedraogo, ${ }^{4}$ \\ Boubacar Nacro, ${ }^{1}$ Georges Moyen5 \\ 1Department of Pediatrics, Sourô Sanou \\ University Hospital, Bobo-Dioulasso, \\ Burkina Faso; ${ }^{2}$ Virology Laboratory of \\ Centre Muraz, IRSS/DRO, Bobo- \\ Dioulasso, Burkina Faso; 3Department \\ of Pediatrics, Régional Hospital of \\ Ouahigouya, Burkina Faso; ${ }^{4}$ Laboratory \\ Department, Sourô Sanou University \\ Hospital, Bobo-Dioulasso, Burkina Faso; \\ 5 University of Brazzaville, Republic of \\ the Congo
}

\begin{abstract}
Viral hepatitis B is a public health issue. We establish the children serological profile of hepatitis B in Bobo-Dioulasso, six years after the introduction of hepatitis B vaccine into the Expanded Program on Immunization. This was a descriptive study of prospective data collection carried out in the Department of Pediatrics and the laboratory of virology of the Centre MURAZ of Bobo-Dioulasso between March 2013 and May 2013. Blood samples were made in search of the following hepatitis B serological markers: anti-HBcAb total, $\mathrm{HBsAg}, \mathrm{Ac}$ anti-HBs, HBeAg, AcHBs, IgM anti-HBc total. The ELISA method with the Monolisa BIORAD reagents was used. A total of 2015 children were included, 1026 (50, 9\%) boys and 989 (49.1\%) girls, at an average age of $58 \pm 48$ months. Out of these 2015 children, $53(2.6 \%)$ were positive to HBsAg including 19 vaccinated cases, one child has received 3 doses plus 1 booster dose of hepatitis $B$ vaccine. We found no statistically significant difference in the carriage of serologic markers of hepatitis B between the unvaccinated group and the vaccinated group. Large-scale studies should be carried out in Burkina Faso to see the real impact of vaccination on the health of our populations.
\end{abstract}

\section{Introduction}

The hepatitis B virus (HBV), part of the hepadnaviridae family, is a small genome of partially double strandled, partially single strangled circular DNA. The markers of infection with the virus are the "s" antigens (HBsAg), they are the surface antigens, the "c" antigens ( $\mathrm{HBcAg})$ and the "e" ( $\mathrm{HBeAg})$ which are both antigens found in the nucleocapsid Core Viral hepatitis B remains a major public health problem. It is one of the leading causes of morbidity and mortality worldwide, 1 particularly in developing countries. A chronical infection with HBV can generate liver failure, cirrhosis, hepatocellular carcinoma. According to WHO, in 2015, the global prevalence of HBV was estimated at $3.5 \%$ in the general population, including 257 million chronic carriers and 887220 deaths attributable to complications. ${ }^{2}$ In sub-Saharan Africa, recognized as a zone of high endemicity, its prevalence is 8 to $20 \%, 3$ and there are an estimated 750 million people living and infected with the hepatitis B virus (HBV), 65 million of whom live with a chronic form. ${ }^{4,5}$ In children, the transmission of HBV is often vertical mainly perinatal; 3 in this case, risk reaches $90 \%$ for children born to mothers carrying the antigen $(\mathrm{HBe} \mathrm{Ag})$. It remains silent during childhood but increases the risk of cirrhosis and liver cancer in adulthood. ${ }^{6}$ The effectiveness of its eradication by a proven systematic vaccination policy already adopted by several countries is the best weapon to reduce the morbidity. Burkina Faso, a country with a prevalence of more than $8 \%, 7$ introduced in 2006 the Hepatitis B vaccine in its Expanded Program on Immunization (EPI) for newborns from 8 weeks of age. The aim of our study is to establish the serological mapping of hepatitis B in children at the Pediatrics Department of Sourô Sanou University Hospital Bobo-Dioulasso, six years after the introduction of hepatitis $B$ vaccine into the EPI.

\section{Materials and Methods}

It was a descriptive study named Cohort Pediatrics Hepatitis-B Bobo (CoPeHeBo) with prospective data collections, conducted between March 2012 and May 2013. The study was conducted in the Department of Pediatrics of University Hospital Sanou Sanou (CHUSS), a hospital-level III and the laboratory of the MURAZ Center of BoboDioulasso. The study population consisted of children aged 1 month to 16 years, seen outpatient and/or hospitalized or for any
Correspondence: Makoura Barro, Sourô Sanou University Hospital, Bobo-Dioulasso, Pediatrics Department

01, BP 676 Bobo 01, Burkina Faso.

Tel.: +22670544470 .

E-mail: barro.mak@fasonet.bf

Key words: Hepatitis B, prevalence, children, serological profile, Bobo-Dioulasso.

Acknowledgments: This study was conducted thanks to the National Agency for AIDS and Viral Hepatitis Research (ANRS).

Contributions: BM did the literature research, and wrote the first draft and the final of the manuscript. VD realized all the tests of hepatitis B, wrote the section of materials and methods. SB and CS did the literature research, and wrote the first draft of the manuscript. OABI and OSA collected clinical data for this manuscript and did also the literature research. OAS realized tests of hepatitis B and gave partial data. MP, NB wrote the protocol of this study. NB and MG reviewed the final manuscript before submission.

Conflict of interest: The authors declare no conflict of interest.

Funding: National Agency for AIDS and Viral Hepatitis Research (ANRS).

Received for publication: 17 July 2019.

Revision received: 3 October 2019.

Accepted for publication: 4 October 2019.

This work is licensed under a Creative Commons Attribution NonCommercial 4.0 License (CC BY-NC 4.0).

(C) Copyright: the Author(s), 2019

Licensee PAGEPress, Italy

Pediatric Reports 2019; 11:8248

doi:10.4081/pr.2019.8248

other reason. Counseling was done with parents to explain the purpose of the study to obtain their informed consent. Children from 1 month to 16 years of consenting parents were included. A questionnaire was intended for collecting sociodemographic data. For the serological analyses, the samples were taken at the CHUSS Pediatrics Day hospital. The different plasmas were recovered after centrifugation in the CHUSS laboratory and then sent to the MURAZ laboratory, respecting the cold chain to be treated. Serological diagnosis of HBV was performed by the ELISA method with BIORAD MONOLISA reagents. The biological markers sought in the plasma were: anti-HBc, HBsAg, anti-HBs, IgManti-HBc, HBeAg / anti-HBe . Study variables were of two types: socio-demograph- 
ic: age, gender, vaccination history, and biological: $\mathrm{HBsAg}, \mathrm{HBeAg}$, anti-HBe, antiHBct, anti-HBs, IgM anti HBc.

\section{Operational definitions}

The presence of HBsAg indicates that the person is infectious. The presence of anti-HBs is generally interpreted as indicating recovery and immunity from hepatitis B virus infection. The presence of anti-HBc indicates previous or ongoing infection with the hepatitis B virus in an undefined time frame. IgM antibody to hepatitis B core antigen (IgM anti-HBc): Positivity indicates recent infection with hepatitis B virus ( $<6$ months). HBeAg is the indirect marker of viral replication; $\mathrm{HBeAb}$ is the HBV marker that controls seroconversion of $\mathrm{HBeAg}$ and thus stops viral replication. Samples whose optical densities had remained in an interval where their values had not reached the positive standard, although above the negative norm, were said to be questionable. ${ }^{8}$

\section{Test statistical test}

The data were entered on the Excel software and analyzed by the STATA software, XLSTAT 2015.1.01.

\section{Ethical consideration}

The study was carried out within the framework of the ANRS 12191 project which received a favorable opinion from the Ethics Committee for Health Research of Burkina Faso for its implementation.

\section{Results}

\section{Immunization status of patients}

The study included 2015 children aged 58 months \pm 48.82 (2 weeks and 186 months). These were 1026 (50.9\%) male children and 989 (49.1\%) female: sex ratio 1.03. Of the 2015 children, $729(36.2 \%)$ were vaccinated and $1286(63.8 \%)$ unvaccinated against HBV (Table 1).

\section{Impact of the vaccine on the car- riage of different markers of $\mathrm{HBV}$}

Of the 2015 children included, HBsAg was positive in 53 cases $(2.62 \%)$ including 28 children sex female. The prevalence of HBsAg carriage was $2 \%$ in age groups 84 to 168 months. Nineteen cases of hepatitis have been reported among vaccinated children, including 11 cases in viral replication. Among these 19 vaccinated cases, 1 child received 3 doses plus 1 booster dose of hepatitis B vaccine Figure 1. We found fewer children with HBsAg in the vaccinated group compared to the unvaccinated group $(\mathrm{n}=34)$. But the difference observed between these 2 groups is not significant; $\mathrm{P}=0.9 ; \mathrm{RR}=1.01$ (95\% CI 0.7-1.45) (Table 2).

\section{Discussion}

Virological tools useful for the diagnosis, monitoring and therapeutic management of HBV-related viral hepatitis are both serological and molecular. ${ }^{1}$ Among serolo gical markers, HBsAg is a good marker for assessing HBV carriage in a population. Its presence is indicative of either acute viral hepatitis B or chronic carriage. 7 The prevalence of carriage of HBsAg in children in our study is $2.63 \%(53 / 2015), 6$ years after the introduction of hepatitis $\mathrm{B}$ vaccine into the EPV. This prevalence is similar to that reported by Abera in Ethiopia (2\%) and lower than that of Eizelo in Nigeria (11.5\%). ${ }^{9,10}$ Higher prevalences are reported by Utsumi in Indonesia, ${ }^{11}$ Qamer in India, ${ }^{12}$ Melendez in Mexico City, ${ }^{13}$ with $3.1 \%$, $4.35 \%$ and $3.1 \%$, respectively. Rates lower than ours are reported by Jooste and Telatela. ${ }^{14,15}$ The differences observed are related to the methodologies used and especially the characteristics of the study population. The HBsAg positive rate among those

Table 1. Immunization status of patients by age group.

\begin{tabular}{lccc}
\hline Age class (months) & Vaccinated, $\mathbf{n}(\%)$ & Unvaccinated, $\mathbf{n}(\%)$ & Total, $\mathbf{n}(\%)$ \\
$0-12$ & $212(10.5)$ & $173(8.6)$ & $385(19.1)$ \\
$13-24$ & $103(5.1)$ & $109(5.4)$ & $212(10.5)$ \\
\hline $25-36$ & $83(4.1)$ & $109(5.4)$ & $192(9.5)$ \\
$37-48$ & $67(3.3)$ & $77(3.8)$ & $144(7.14)$ \\
\hline $49-60$ & $76(3.8)$ & $91(4.5)$ & $167(8.28)$ \\
$61-72$ & $39(1.9)$ & $89(4.4)$ & $128(6.35)$ \\
\hline $73-84$ & $16(0.8)$ & $93(4.6)$ & $109(5.4)$ \\
$85-96$ & $31(1.5)$ & $109(5.4)$ & $140(6.9)$ \\
\hline $97-108$ & $20(1)$ & $81(4.02)$ & $101(5.01)$ \\
$108-120$ & $23(1.1)$ & $93(4.6)$ & $116(5.7)$ \\
\hline$>120$ & $59(2.9)$ & $262(13)$ & $321(15.9)$ \\
Total & $729(36.2)$ & $1286(63.8)$ & $2015(100)$ \\
\hline
\end{tabular}

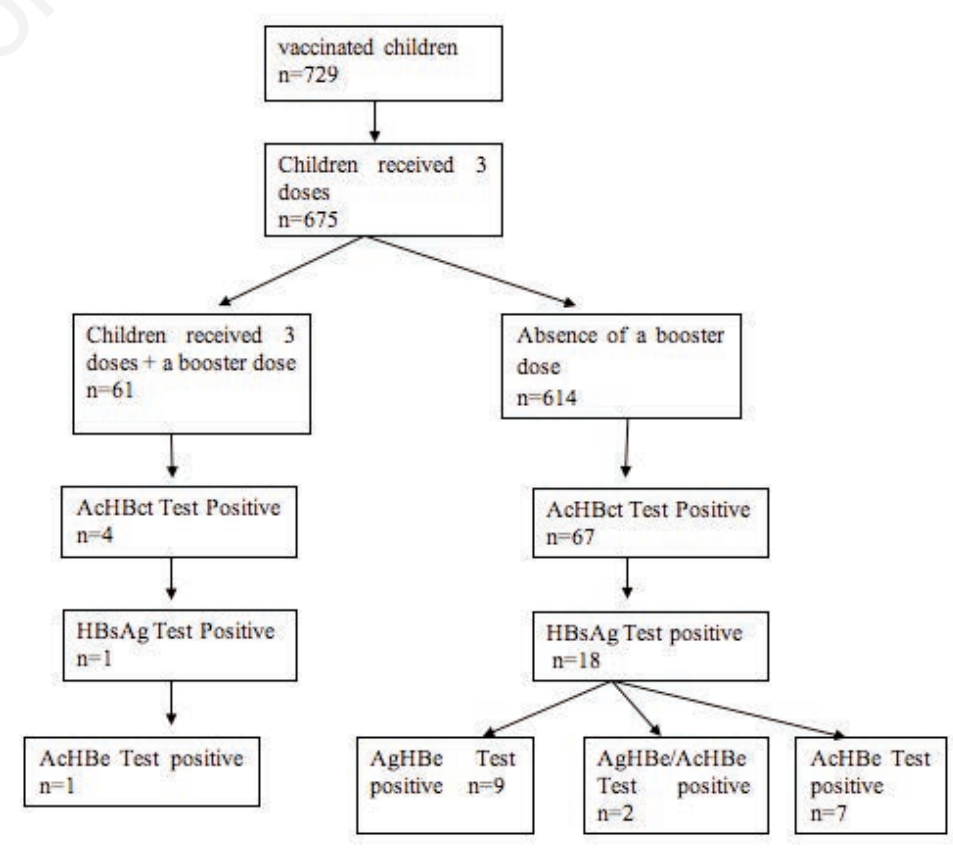

Figure 1. Serological markers according to the number of booster doses of vaccination against hepatitis $B$. 
Table 2. Impact of the vaccine on the carriage of different markers of HBV.

\begin{tabular}{ccccc} 
Variables & Effective, tot & Vaccinated, n (\%) & Not vaccinated, n (\%) & P-value \\
Anti-HBc & 2011 & & & 0.33 \\
Negative & & $650(32.3)$ & $1128(56.1)$ & \\
Positive & & $77(3.8)$ & $156(7.7)$ & 0.92 \\
HBsAg & 2015 & $710(35.2)$ & $1252(62.2)$ & \\
Negative & & $19(0.9)$ & $34(1.7)$ & 0.82 \\
Positive & & & $1211(60.1)$ & \\
\hline Anti-HBs & 2015 & $689(34.2)$ & $75(3.7)$ & 1 \\
Negative & & $40(2)$ & $1283(63.7)$ & \\
Positive & & $728(36.1)$ & $3(0.1)$ & \\
IgM anti-HBc & 2015 & $1(0.05)$ & & \\
Negative & & & $1260(65.5)$ & \\
Positive & & $718(35.6)$ & $26(1.3)$ & \\
\hline HBeAg & 2015 & $11(0.5)$ & \\
Negative & & & \\
Positive & & &
\end{tabular}

vaccinated was $0.9 \%$, much lower than that in unvaccinated group (1.7\%) and this may be partly because of the effect of introduced in 2006 the Hepatitis B vaccine in the Expanded Program on Immunization (EPI) of Burkina Faso. But the difference observed between these 2 groups is not significant $\mathrm{P}=0.92$. The positivity of HBsAg in the vaccinated group is explained by the fact that the administration of a series of three doses of hepatitis B vaccine does not induce protective levels of antibodies against HBsAg (anti-HBs) at 100\%.16 However, for better protection, the literature recommends the administration of the first dose of the vaccine at birth. ${ }^{16,17}$ In Burkina Faso, children are vaccinated late, with the first dose administered at 8 weeks of age. This situation challenges our countries to change their immunization policy and implement the WHO recommendations in order to prevent HBV mother-to-child transmission. ${ }^{17}$ Through this study, we also recommend booster doses for better efficacy.

\section{Conclusions}

The prevalence of HBsAg in the pediatric department of CHUSS BoboDioulasso, is high. Cases of hepatitis have been reported despite vaccination. It is crucial to increase immunization coverage by the administration of the first dose of the vaccine at birth and booster doses. Largescale studies should be carried out in Burkina to see the real impact of vaccination on the health of our populations.

\section{References}

1. Spearman CW, Afihene M, Ally R et al. Hepatitis B in sub-Saharan Africa: strategies to achieve the 2030 elimination targets. Lancet Gastroenterol Hepatol 2017;2:900-9.

2. OMS. Hépatite B. Available from: https://wwwwhoint/fr/news-room/factsheets/detail/hepatitis-b. 2015. Consulté le 23 Novembre 2018

3. Njoya CF, Nkwabong E, Njoya O, et al. Facteurs Associés à la Transmission du Virus de l'Hépatite $\mathrm{B}$ de la Mère à l'Enfant: une enquête CAP. Health Sci Dis 2018;19:32-5.

4. Malam-Abdou B, Brah S, Chefou ME, et al. Le risque infectieux post-transfusionnel: une étude comparative sur la séroprévalence du VIH, des hépatites B et $\mathrm{C}$ et de la Syphilis: à propos de 202 patients testés à l'Hôpital National de Niamey (HNN). Health Sci Dis

5. Loriette M, Birguel J, Damza R et al. Une expérience de lutte contre l'hépatite $\mathrm{B}$ en zone rurale à l'extrême nord du Cameroun. Méd Santé Tropical 2015; 25:422-7.

6. Broderick AL, Jonas MM. Hepatitis B in children. Seminars in liver disease. Thieme Medical Publishers, Inc., 2003.

7. Sidibe S, Youssoufi Sacko B, Traoré I. Prévalence des marqueurs sérologiques $\mathrm{du}$ virus de l'hépatite $\mathrm{B}$ chez les femmes enceintes dans le district de Bamako, Mali. Bull Soc Pathol Exot 2001;94:339-41.

8. MemoBio. Virus de l'hépatite B (VHB). 2016;17:1-4
Available from: http://wwwmemobiofr/html/viro/vi_vhb_sehtml. Consulté le 23 Novembre 2018

9. Abera B, Zenebe Y, Mulu W, et al. Seroprevalence of hepatitis B and C viruses and risk factors in HIV infected children at the felgehiwot referral hospital, Ethiopia. BMC Res Notes 2014;7:838.

10. Ezeilo MC, Engwa GA, Iroha RI, Odimegwu DC. Seroprevalence and Associated Risk Factors of Hepatitis B Virus Infection Among Children in Enugu Metropolis. Virol Res Treat 2018;9:1-7.

11. Utsumi T, Yano Y, Lusida MI, et al. Serologic and molecular characteristics of hepatitis B virus among school children in East Java, Indonesia. Am J Trop Med Hyg 2010;83:189-93.

12. Qamer S, Shahab T, Alam S et al. Agespecific prevalence of hepatitis B surface antigen in pediatric population of Aligarh, North India. Indian J Pediatr 2004;71:965-7.

13. Escobedo-Meléndez G, Fierro NA, Roman S, et al. Prevalence of hepatitis $\mathrm{A}, \mathrm{B}$ and $\mathrm{C}$ serological markers in children from western Mexico. Ann Hepatol 2012;11:194-201.

14. Jooste P, van Zyl A, Adland E, et al. Screening, characterisation and prevention of Hepatitis B virus (HBV) coinfection in HIV-positive children in South Africa. J Clin Virol 2016;85:71-4.

15. Telatela SP, Matee MI, Munubhi EK. Seroprevalence of hepatitis B and C viral co-infections among children infected with human immunodeficiency virus attending the paediatric HIV care and treatment center at Muhimbili National Hospital in Dar-es-Salaam, Tanzania. BMC Public Health 2007; 7:338.

16. WHO. Impact de la vaccination contre l'hépatite B: meilleures pratiques pour mener une enquête sérologique. WHO/IVB/11.08F 2014. Available from: https://apps.who.int/iris/bitstream/handle/10665/112854/WHO IV B_11.08_fre.pdf; jsessionid $=205 \mathrm{~F} 2 \mathrm{~F} 91$ C212AF8AA2611CB16DECB4B0?seq uence $=1$. Accessed September 20, 2019

17. WHO. Immunization, Vaccines and Biologicals. 2018. vailable from: https://www.who.int/immunization/diseases/hepatitisB/en/. Accessed August 11, 2019. 